\title{
Educación geográfica: resultados y experiencias sobre la pesquisa documental
}

Lic. Katherine Dayanna Arévalo Suárez, Colombia.

https://orcid.org/0000-0002-5001-6639

kdarevalos@correo.udistrital.edu.co

Lic. Daisy Carolina Sánchez Sánchez, Colombia.

https://orcid.org/0000-0003-0103-0546

dacsanchezs@correo.udistrital.edu.co

Dra. Liliana Angélica Rodríguez Pizzinato, Colombia.

larodriguezp@udistrital.edu.co

https://orcid.org/0000-0003-1414-936X

Universidad Distrital Francisco José de Caldas, Colombia.

Recibido: 24 de junio del 2020

Aceptado: 25 de agosto del 2020

\section{Resumen}

El artículo presenta el resultado parcial de una investigación documental que toma aportes del Proyecto de Difusión del programa de Geografía y acompañamiento a colegios 2016-2022 Diagnóstico y propuesta de formación en Geografía para la educación básica y media de la Universidad Externado de Colombia y del Ciclo de innovación de la Licenciatura en Educación Básica con Énfasis en Ciencias Sociales de la Universidad Distrital Francisco José de Caldas (Bogotá, Colombia), en el marco de la línea de investigación Educación geográfica, formación docente y ciudadana. Se aborda la relación entre educación y geografía para construir un estado de conocimiento particular en la indagación de la categoría de educación geográfica, con la intención de identificar desarrollos conceptuales, pedagógicos y metodológicos sobre la alfabetización espacial de escolares o ciudadanos y presentar los ejes analíticos reconocidos desde la pesquisa documental realizada. Además, este proceso vincula un ejercicio formativo de ingreso para nuevos miembros al Semillero de Investigación Formación y Educación Geográfica Itinerantes, con un trabajo diseñado por el mismo denominado Esquema de acompañamiento uno a uno, caracterizado por un ejercicio tutorial focalizado por parte de los miembros de base del semillero, en el cual además de aportar sus experiencias y saberes, aprenden a orientar un proceso y producir conocimiento en este caso sobre la relación entre geografía y educación. La actividad mencionada se asume como una parte importante en el fortalecimiento de habilidades, destrezas y competencias pedagógicas de los futuros docentes en ciencias sociales. Por otra parte, el artículo presenta algunas de las tendencias identificadas a nivel teórico y procedimental sobre las discusiones actuales frente a la educación geográfica, 
utilizando como metodología la estrategia de mapeamiento informacional bibliográfico con la elaboración de matrices de resumen y contenido para el registro y tematización de las fuentes consultadas. Así mismo, estos resultados permiten establecer semejanzas y diferencias en la revisión realizada en los dos proyectos; reconocer lo que se ha dicho frente a la categoría de educación geográfica y reflexionar la experiencia del proceso vivido como docentes en formación, sujetos y ciudadanos.

Palabras clave: Educación Geográfica, mapeamiento informacional bibliográfico, formación inicial docente, práctica pedagógica.

\section{Geographical education: results and experiences on documentary research}

\section{Abstract}

The article presents the partial result of a documentary research that takes contributions from the Project of Dissemination of the Geography program and accompaniment to schools 20162022 - Diagnosis and proposal of training in Geography for basic and middle education of the Universidad Externado de Colombia and the Cycle Innovation of the Bachelor's Degree in Basic Education with Emphasis in Social Sciences of the Universidad Distrital Francisco José de Caldas (Bogotá, Colombia), within the framework of the research line Geographical education, teacher and citizen training. The relationship between education and geography is addressed to build a particular state of knowledge in the research of the category of geographical education, with the intention of identifying conceptual, pedagogical and methodological developments on the spatial literacy of schoolchildren or citizens and presenting the analytical axes recognized from the documentary research carried out. In addition, this process links a training exercise of entry for new members to the hotbed of research Training and Geographical Education Itinerant, with a work designed by it called The Accompaniment Scheme one by one, characterized by a tutorial exercise focused by the base members of the seedbed, in which in addition to contributing their experiences and knowledge, they learn to guide a process and produce knowledge in this case about the relationship between geography and education. The above-mentioned activity is assumed as an important part in strengthening the skills pedagogical of future teachers in social sciences. Furthermore, the article presents some of the trends identified at the theoretical and procedural level on current discussions against geographical education, using as a methodology the strategy of bibliographic informational mapping with the elaboration of summary and content matrices for the registration and 
theorization of the sources consulted. Likewise, these results allow to establish similarities and differences in the review carried out in the two projects; recognize what has been said in the face of the category of geographical education and reflect on the experience of the process lived as teachers in training, subjects and citizens.

Keywords: Geographical Education, bibliographic informational mapping, initial teacher training, pedagogical practice.

\section{Aproximaciones metodológicas}

La relación entre educación y geografía es una preocupación importante en los procesos de formación inicial del docente para el fortalecimiento de habilidades, destrezas y competencias pedagógicas que le permiten la comprensión de la realidad espacial y ciudadana en diferentes niveles escolares, que además se vincula con el desarrollo académico en la práctica pedagógica y la investigación educativa en la profesión docente.

El artículo es un resultado parcial de una investigación documental interinstitucional que busca construir un estado de conocimiento de la relación mencionada. La fuente principal de análisis es el documento, sobre el que se realiza una decodificación mediante la identificación de citas procesadas, por medio de unidades analíticas que permiten otras miradas de acuerdo con los resultados que la lectura especializada propone.

La metodología empleada en la pesquisa documental es la de mapeamiento informacional bibliográfico propuesto por André (2009), cuyo proceso significa un ejercicio de síntesis, que requiere de una lectura atenta de las informaciones, su comprensión, la identificación de las ideas principales del autor y su registro escrito de modo conciso; además se constituye en una opción para orientar búsquedas, para seleccionar fuentes bibliográficas y determinar el desarrollo conceptual de perspectivas de investigación.

Esta estrategia presenta dos fases de desarrollo; la primera corresponde a una matriz de resumen en la cual se sintetiza el proceso de búsqueda a partir de la estructura de la Tabla 1. En esta fase son tres los criterios de selección de fuentes documentales para el Proyecto de difusión: el primero responde a publicaciones realizadas entre 1994 y 2018, el segundo a localizar publicaciones en Colombia, América Latina y España, por último, se establece la revisión en escenarios académicos especializados en temas geográficos y de educación geográfica. 
Tabla 1. Estructura base matriz de resumen

\begin{tabular}{|c|c|c|c|c|c|c|c|}
\hline $\begin{array}{c}\text { N. } \\
\text { Documento }\end{array}$ & $\begin{array}{c}\text { Referencia } \\
\text { Bibliográfica }\end{array}$ & $\begin{array}{c}\text { Tipo De } \\
\text { Documento }\end{array}$ & $\begin{array}{c}\text { Sitio De } \\
\text { Publicación }\end{array}$ & Autor & Volumen & $\begin{array}{c}N^{\circ} \text { De } \\
\text { Revista }\end{array}$ & Año \\
\hline
\end{tabular}

\begin{tabular}{|c|l|l|c|l|l|l|}
\hline $\begin{array}{c}\text { Título } \\
\text { Documento }\end{array}$ & Resumen & Categoría & $\begin{array}{c}\text { Subcategorías } \\
- \\
\text { Estandarizadas }\end{array}$ & Responsable & Comentarios & $\begin{array}{c}\text { Ejes } \\
\text { Temáticos O } \\
\text { Subcategorías }\end{array}$ \\
\hline
\end{tabular}

Fuente: Elaboración propia con base en los proyectos

Por otra parte, el Ciclo de innovación adopta los siguientes criterios de selección de fuentes documentales: idioma de circulación (que debe ser español o portugués) y escala geográfica nacional o internacional. De acuerdo a lo anterior, la revisión se hace en repositorios documentales en los que se ubican monografías, revistas universitarias y científicas relacionadas con educación, geografía, didáctica de la geografía o afines a estas; además de consultar las memorias de los Encuentros de Geógrafos de América Latina y proyectos como Nós Propomos, que plantea una lectura de contexto próximo para proponer soluciones por parte de los escolares a problemas espaciales identificados.

Posteriormente, se establecen categorías y subcategorías de análisis que se consolidan en discusiones, validaciones y acuerdos por parte de los miembros en los proyectos del Programa de difusión de la Universidad Externado y del Ciclo de innovación de la Universidad Distrital. Dichas unidades analíticas permiten tematizar y orientar el contenido de los resúmenes, para luego abordar el procesamiento en extenso de fuentes, que se realiza en una matriz de contenido que conserva de base la estructura de la matriz de resumen hasta la casilla de Título de documento y se complementa con los campos que posibilitan la tematización de las citas textuales identificadas (Tabla 2). 
Tabla 2. Estructura matriz de contenido

\begin{tabular}{|c|c|c|c|c|c|}
\hline $\begin{array}{c}\text { Título } \\
\text { Documento }\end{array}$ & Citas & Comentarios & Categorías & Subcategorías & $\begin{array}{c}\text { Responsable } \\
\text { Revisión Extenso }\end{array}$ \\
\hline
\end{tabular}

Fuente: Elaboración propia con base en los proyectos

Este proceso de tematización es flexible en tanto permite identificar categorías emergentes, que aparecen producto de la interpretación de los resultados de las investigaciones consultadas y que son validadas de igual manera. El mapeamiento bibliográfico contribuye a clarificar el horizonte epistemológico sobre educación y geografía en relación con la organización de los documentos, estructuras conceptuales y definiciones propuestas en los mismos. Así como tendencias, permanencias o ausencias investigativas frente a las relaciones entre estos campos, aportando en un ejercicio escritural más claro y consistente que brinda la posibilidad de comunicar resultados desde una perspectiva de hallazgos y de estado de conocimiento de lo estudiado.

\section{Ruta de trabajo: Unidades de análisis sobre educación geográfica}

La educación geográfica es una categoría en la que converge el interés de los proyectos mencionados, la cual se refiere al campo de reflexión y acción que vincula a la geografía académica, escolar y experiencial y favorece que los sujetos sociales, los ciudadanos, desarrollen habilidades de pensamiento espacial, necesarias en escenarios académicos y en sus prácticas de la vida cotidiana. Así, ésta no se encuentra circunscrita solamente al ámbito de la escuela formal, sino que se expresa en distintos ámbitos en los que se usa y emplea conocimiento geográfico para comprender y resolver problemas tanto locales y cercanos, como de gestión y administración del espacio-territorio en las escalas local, regional, nacional y mundial.

Sin embargo, se encuentra una diferencia en la asignación de subcategorías de cada proyecto de acuerdo a las intenciones investigativas en las que enfatiza cada uno (Gráfico 1). Las 
subcategorías consideradas en el Proyecto de difusión corresponden a geografía escolar, alfabetización espacial, formación de docentes en ciencias sociales y/o geografía, normatividad educativa, educación ambiental, formación ciudadana y textos escolares. En el caso del Ciclo de innovación son educación ambiental, educación comunitaria, habilidades y destrezas geográficas.

Gráfico 1. Presentación subcategorías por proyecto

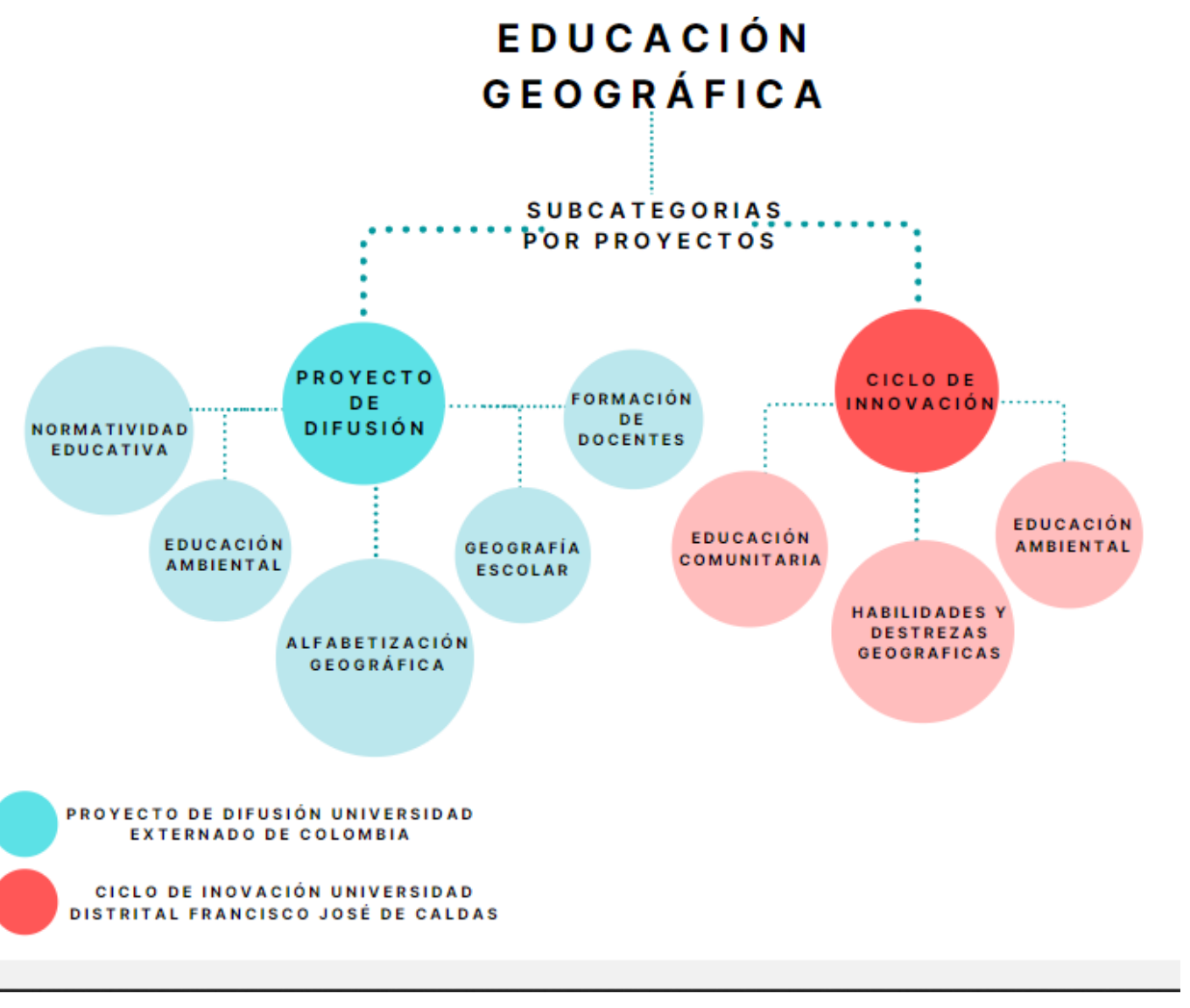

Fuente: Elaboración propia basada en tabla de definiciones proyectos interinstitucionales

Las definiciones de estas subcategorías posibilitan el reconocimiento de algunas relaciones y tendencias a nivel conceptual, epistemológico y metodológico cuyo anclaje se propone en torno al desarrollo de pensamiento geográfico, contextos escolares o sociales, 
formación de educadores y lineamientos curriculares, la cuales constituyen la síntesis de la convergencia en las definiciones de los dos proyectos. (Tabla 3).

Tabla 3. Definición subcategorías por unidades de análisis

\begin{tabular}{|c|l|}
\hline $\begin{array}{c}\text { Tendencias teórico } \\
\text { empíricas }\end{array}$ & \multicolumn{1}{|c|}{ Síntesis conceptual } \\
\hline $\begin{array}{c}\text { Desarrollo de pensamiento } \\
\text { geográfico }\end{array}$ & $\begin{array}{l}\text { Se refiere a las acciones, relaciones intencionadas y capacidades } \\
\text { que los sujetos sociales establecen dentro o fuera de las } \\
\text { instituciones educativas estas permiten desarrollar } \\
\text { conocimientos, destrezas, habilidades, actitudes y valoraciones } \\
\text { espaciales tales como ubicación, situación, graficidad, } \\
\text { visualidad, medición y apropiación espacio temporal para } \\
\text { interactuar en prácticas cotidianas en un mundo multi-escalar. } \\
\text { Estas pueden incluir reflexiones, prácticas pedagógicas, } \\
\text { didácticas, y experienciales. }\end{array}$ \\
\hline Contextos escolares $o$ & $\begin{array}{l}\text { Se refieren al conjunto de circunstancias, factores, } \\
\text { conocimientos, prácticas e identidades que se gestan alrededor } \\
\text { de sujetos, comunidades, procesos de socialización, educación y } \\
\text { formación en determinadas condiciones de vida o trabajo en la } \\
\text { cotidianidad. }\end{array}$ \\
\hline Formación de educadores y y \\
lineamientos curriculares & $\begin{array}{l}\text { Se refieren al interés por conocer la relación entre las } \\
\text { orientaciones epistemológicas, conceptuales, metodológicas y } \\
\text { prácticas, presentes en la formación académica y profesional de } \\
\text { docentes, en torno al conocimiento y educación geográfica. Se } \\
\text { interesa por conocer los aportes, vacíos o contradicciones que } \\
\text { tienen los docentes de acuerdo a la reglamentación y políticas } \\
\text { educativas que tendrán incidencia en su trabajo o práctica } \\
\text { profesional. }\end{array}$ \\
\hline
\end{tabular}

Fuente: Elaboración propia basada en tabla de definiciones proyectos interinstitucionales

La exploración de las matrices de resumen producidas en los proyectos interinstitucionales coinciden con mayor relevancia en la tendencia de desarrollo de pensamiento geográfico, ya que “el análisis, interpretación y explicación del espacio geográfico se basa en temas articuladores como localización, lugar, interacción hombre-medio, movimientos y regiones", (Mercado y Moore, 1997, p. 1), es decir, que el desarrollo de pensamiento espacial posibilita desde la observación, descripción y explicación, comprender y actuar de manera consecuente y propositiva sobre el entorno.

De la misma manera, los proyectos insisten en el potencial que se puede aprovechar en la escuela, para avanzar en procesos de alfabetización espacial en el que el ambiente educativo 
juega un papel fundamental en la formación del estudiante y le permite hacer visibles componentes, actores, fenómenos, situaciones que se inscriben en el espacio y definen formas de organización social distintas en el mismo (Rodríguez, Pérez y Rodríguez, 2003).

Las tendencias de contextos escolares o sociales, formación de educadores y lineamientos curriculares ocupan el segundo y tercer lugar respectivamente. Se precisan componentes que destacan la educación geográfica como campo de acción y reflexión, en tanto es posible construir una geografía escolar según Córdoba (2005), articulada a problemas contemporáneos, en la cual el lugar y lo local se convierten en una alternativa pedagógica fundamental para el docente; o como manifiesta Pastrán (2007) desde escenarios con comunidades rurales y fronterizas, en los cuales la educación geográfica ofrece posibilidades de estudio, descripción, análisis e interpretación para la formulación de opciones y acciones que respondan a sus necesidades.

De acuerdo a lo anterior, se hace necesario que la educación geográfica disminuya la resistencia al cambio innovador y facilite la enseñanza geográfica ante las necesidades de la sociedad actual. En ese sentido, Santiago (2013) al centrar la atención en el aula de clase, encuentra vigente la enseñanza geográfica decimonónica alrededor de una práctica escolar cotidiana, meramente informativa de contenidos programáticos geográficos, que se desvinculan del mundo inmediato, el contexto y la experiencia de los escolares.

Es importante entonces, el reconocimiento del papel docente como plantea Rodríguez y Von (2008) al presentarlos como mediadores culturales encargados de establecer enlaces entre el conocimiento empírico y el conocimiento escolar. Es necesario señalar que la educación geográfica no solo se instala en el marco escolar, sino en los múltiples contextos sociales en los que están inmerso el sujeto y el ciudadano.

\section{Resultados teóricos de la investigación documental}

La segunda fase de la estrategia de mapeamiento informacional bibliográfico corresponde al procesamiento en extenso de las fuentes consultadas, en este punto se realiza una lectura detallada de los documentos, en base a las subcategorías que están preestablecidas; en relación con la categoría abordada en los proyectos sobre educación geográfica se encuentran 698 documentos de diferente tipo (Tabla 4). 
Así, se identifica los mayores aportes en artículos de revista con un 64,05\% para el ciclo de innovación y ponencias para el Proyecto de difusión con un 50,26\%, es decir, que estas fuentes representan cerca del $70 \%$ en el total de la documentación revisada. La suma de capítulos de libro y monografías esta alrededor del 30\% y los libros y tesis doctorales tiene la menor presencia con cerca del $3 \%$.

Tabla 4. Tipo de documento por proyecto

\begin{tabular}{|c|c|c|}
\hline \multicolumn{3}{|c|}{ Proyecto Difusión } \\
\hline $\begin{array}{c}\text { Tipo de } \\
\text { documento }\end{array}$ & Cantidad & Porcentaje \\
\hline $\begin{array}{c}\text { Artículo de } \\
\text { revista }\end{array}$ & 65 & $34,39 \%$ \\
\hline Capítulo de libro & 25 & $13,23 \%$ \\
\hline Libro & 3 & $1,59 \%$ \\
\hline Ponencia & 95 & $50,26 \%$ \\
\hline Tesis doctoral & 1 & $0,53 \%$ \\
\hline Suma total & \multicolumn{2}{|c|}{189} \\
\hline
\end{tabular}

\begin{tabular}{|c|c|c|}
\hline \multicolumn{3}{|c|}{ Ciclo Innovación } \\
\hline $\begin{array}{c}\text { Tipo de } \\
\text { documento }\end{array}$ & Cantidad & Porcentaje \\
\hline $\begin{array}{c}\text { Artículo de } \\
\text { revista }\end{array}$ & 326 & $64,05 \%$ \\
\hline Capítulo de libro & 41 & $8,06 \%$ \\
\hline Monografía & 102 & $20,04 \%$ \\
\hline Ponencia & 38 & $7,47 \%$ \\
\hline Tesis doctoral & 2 & $0,39 \%$ \\
\hline Suma total & \multicolumn{2}{|c|}{509} \\
\hline
\end{tabular}

Fuente: Elaboración propia con base en los proyectos interinstitucionales

Frente al sitio de publicación y de acuerdo a los criterios de selección se encuentra de manera común que, para el Proyecto de difusión y el Ciclo de innovación, Colombia es el país que aporta la mayor cantidad de documentos, estos responden a publicaciones en revistas académicas y científicas (Gráfico 2) que coinciden con sitios donde se han desarrollado eventos académicos como los Encuentros de Geógrafos de América Latina.

Así mismo, las comunidades académicas en estos sitios realizan investigaciones sobre la educación y geografía no solo en el ámbito escolar, ya que se reconocen una posibilidad importante de un conocimiento geográfico inteligible, próximo y vital que puede aportar en la formación de ciudadanos alfabetizados espacialmente que puedan actuar de manera más consciente, participativa y propositiva en sus entornos de vida. 
Gráfico 2. Sitio de publicación de las fuentes

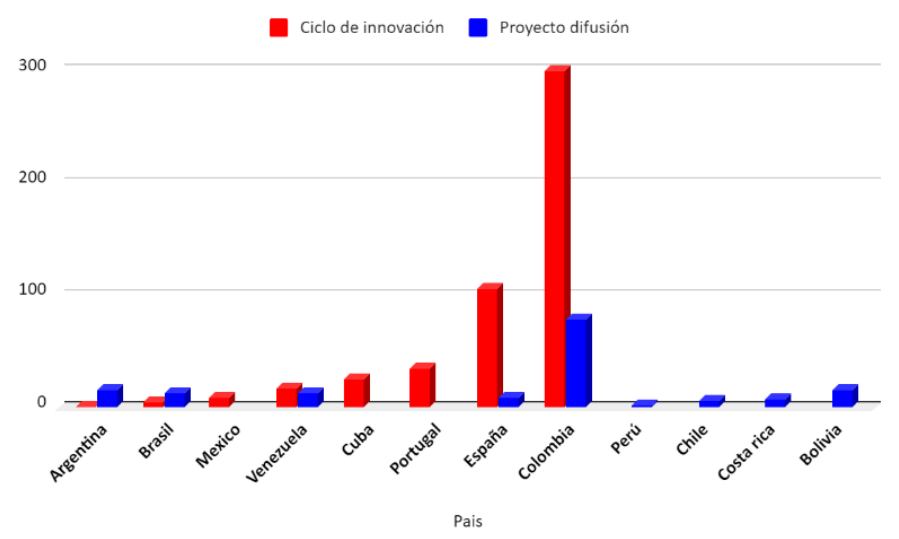

Fuente: Elaboración propia basada en matrices de resumen proyectos interinstitucionales

La estrategia de mapeamiento permite tematizar de manera flexible y conjugar la categoría de educación geográfica con subcategorías propuestas para otras unidades de análisis de la misma jerarquía se aprecian algunas subcategorías emergentes que presentan un estado de validación diferenciado al inicial en los proyectos. Así, la creación de las matrices y su procesamiento en extenso responde en el caso del ciclo de innovación a seis ejercicios investigativos que se encuentran en el marco de la educación geográfica, formación docente y ciudadana, que abordan temáticas como barrio, territorio, migración, vida cotidiana, lúdica y educación ambiental. Esta interrelación epistemológica y conceptual se identifica en la tendencia de desarrollo de pensamiento geográfico y contextos escolares o sociales, pues

el espacio, como objeto de estudio de la geografía, se constituye en la principal fuente de recursos naturales y humanos, haciendo necesario el conocimiento espacial continuo de los contextos socio - territoriales y socio - espaciales. Dicho conocimiento es relevante a nivel social, económico, político y cultural ya que permite acceder al aprendizaje de una geografía que comprende lo regional, local, global, urbano y ciudadano, a partir de una reflexión del espacio, basada en la apropiación y percepción que posee el hombre sobre éste, siendo así viable construir estrategias adecuadas para su conocimiento, interpretación, valoración y utilización acorde con la formación ciudadana (Moreno, 2011, p. 64).

En concordancia con la diversidad de temáticas que se abordan en relación a la categoría de educación geográfica, se evidencia la posibilidad de explicar problemas sociales, históricos 
y cotidianos desde espacios geográficos próximos o lejanos. Para el caso del Proyecto de difusión hay una intencionalidad manifiesta en su eje de investigación, por reconocer en la categoría de educación geográfica indicios que orienten el diagnóstico y propuesta de formación en geografía para la educación básica y media, en ese sentido

el intentar relacionar el saber geográfico con el conocimiento social y la construcción de pensamiento crítico responde en gran medida a la posibilidad que las Ciencias Sociales nos ofrecen de permitir que los estudiantes comprendan los problemas humanos desde la interdisciplinariedad, reflexionando sobre ellos y buscando alternativas de solución en el marco de sus vivencias cotidianas (Rodríguez, Moreno y Cely, 2010, p. 129).

Entonces se entiende que la geografía escolar debería contribuir en la orientación de propuestas que permitan afianzar los vínculos de los estudiantes con su contexto, es decir el barrio, la localidad, los lugares donde transcurre parte sustancial de sus vidas, pues allí se configura su experiencia a partir de la relación que tienen con el espacio. Lo anterior, se relaciona con uno de los aportes de la geografía como lo propone Cabeza (2008), al permitir que ellos construyan un nuevo conocimiento a partir de su experiencia, a fin de lograr que tengan un alto grado de metacognición y describan, analicen y luego interpreten y reflexionen acerca de la realidad que les atañe como ciudadanos en la sociedad.

De este modo, el procesamiento en extenso prevé un abordaje sistemático cuyo estado de validación de unidades de análisis es constante. Así, la estrategia de mapeamiento informacional bibliográfico se configura como una alternativa que da cuenta del estado de conocimiento, un ejemplo de ello es la construcción y presentación de esta ponencia. Vale la pena mencionar el acento de algunas ideas sobre el tono de escritura de los docentes que tiene un carácter propositivo, en el cual la palabra y la teoría permean escenarios prácticos que otorgan mayor validez a la sistematización de experiencias e incluso a la matriz elaborada.

\section{Reconstrucción de la experiencia: un ejercicio formativo}

La reflexión de la experiencia en el proceso de formación inicial docente aparece vinculada alrededor del componente pedagógico del proyecto del ciclo de innovación de la Licenciatura en Ciencias Sociales y del ejercicio académico del Semillero de Investigación Formación y Educación Geográfica Itinerantes, entendiendo que las decisiones docentes están orientadas por 
sus trayectorias académicas y personales.

Los docentes son sujetos antes que profesionales, que en su quehacer pedagógico también expresan sentimientos, actitudes, vivencias y emociones, además de ser conscientes que sus errores pueden ser corregidos (Alliud, 2003), o que sus acciones no siempre tienen el mismo efecto en la actividad educativa. Por ejemplo, en la práctica académica cuando una metodología, taller, actividad o evaluación responde a los objetivos propuestos, no necesariamente significa que sea exitosa en contextos escolares similares.

La reflexión del docente, en formación y en ejercicio, sobre su experiencia debería ser natural, cotidiana y prolongada; sin embargo, las expresiones habituales de su acción se constituyen en prácticas rutinarias o monótonas que no se cuestionan, bajo un supuesto de efectividad certeza, validez o éxito en los procesos de enseñanza. Por ello, colocar las prácticas pedagógicas sobre la lupa de la incertidumbre debería motivar un ejercicio metacognitivo de reflexión en y sobre la acción (Schön, 1992).

Así mismo, la vocación docente es un elemento enriquecedor a nivel personal y profesional, en relación a que "no existe la enseñanza sin vocación" (Alliaud y Antelo, 2011, p. 106). La experiencia como sujetos docentes y el ejercicio que sobre esta posibilita da cuenta de ese límite sobre lo que se sabe a ciencia cierta y lo que no; por lo que es importante señalar que la experiencia que se configura en los espacios de la vida cotidiana, gana protagonismo por su trayectoria académica y profesional. Es por ello, que quizá como docentes no necesariamente se enseña lo que se sabe sino lo que se es.

El interés por la participación en el semillero de investigación mencionado, trasciende la necesidad de afianzar la formación pedagógica y va más allá, al fortalecer algunas habilidades y competencias interpersonales, comunicacionales e investigativas, que con el tiempo se constituyen en condiciones de posibilidad para armonizar lo subjetivo y lo académico en un mismo escenario. En este punto es fundamental el acompañamiento uno a uno con el que se cuenta, pues la experiencia de los pares académicos y quien lidera el proceso, orienta y nutre esa forma en que se pueden cuestionar las acciones, los pensamientos y avanzar sobre una praxis pedagógica y docente.

En el presente ejercicio investigativo se reconocen algunas actitudes y conocimientos que como futuros docentes son importantes a propósito de la experiencia de reflexionar la 
experiencia, como el entusiasmo, inventiva, disposición, interés, intención, saber pedagógico, saber científico y conocimiento del contenido. Lo anterior, en relación directa al quehacer docente que este tiene vinculado o no con sujetos escolares, en la búsqueda de una coherencia personal que oriente su formación inicial.

\section{Consideraciones finales}

Las ideas resultantes del proceso de reflexión de hallazgos parciales, en primer lugar, reconocen la relación conceptual y metodológica que tienen ambos proyectos al hacer uso del mapeamiento informacional bibliográfico y la diversidad de matices que puede tener una misma categoría a la luz de una intencionalidad investigativa diferenciada, lo que configura la posibilidad de diálogo a partir de la comparación y la diferencia.

Este punto también se presenta como invitación a realizar un acercamiento a dicha estrategia y considera el conjunto de tendencias y protocolos de armonización resultado de las diferentes versiones de las matrices como es la experiencia del Semillero de Investigación Formación y Educación Geográfica Itinerantes que ha hecho aportes al planteamiento de André (2009), con criterios asociados con algunos datos de la información bibliográfica como por ejemplo tipo de documento, institución académica y sitio de publicación; así como subcategorías y comentarios.

Por otra parte, la negociación de significados que aporta el semillero frente a la tematización por categorías y subcategorías es una actividad permanente, con una metodología que resulta flexible y puede responder no solo al ejercicio investigativo sino a otros escenarios académicos. En segundo lugar, se destaca la cantidad de fuentes que pueden ser abordadas en un estado de conocimiento, en la suma de ambos proyectos se identifican más de 600 fuentes rastreadas sobre educación geográfica. Se reconoce así mismo, las posibilidades que brinda el trabajo conjunto no solo a nivel de pares académicos sino entre instituciones, ya que los diálogos, discusiones, debates y consensos de quienes participan directa o indirectamente en la 
pesquisa documental amplían el panorama conceptual y de problematización sobre aspectos de interés.

En tercer lugar, el desarrollo de este trabajo reconoce una apuesta formativa del semillero que propone un esquema de observación y acompañamiento focalizado sobre sus líneas de investigación y las que son de interés interinstitucional. De tal manera, los frentes de trabajo constituidos, en particular el que se pregunta por la relación educación y geografía, permite resaltar la vinculación entre pares y quien dirige el proceso para integrar nuevos miembros al Semillero de Investigación Formación y Educación Geográfica Itinerantes con producción académica ligada al ejercicio mencionado.

Finalmente, otro hallazgo destacado corresponde a los esfuerzos que ha hecho la educación geográfica por fomentar experiencias de aprendizaje, como una alternativa en la transformación de prácticas espaciales significativas para docentes e investigadores que sistematizan y publican sus resultados. El tono propositivo bajo el cual los actores anteriores llevan a cabo sus prácticas, es visible en el momento de vincular el territorio como espacio cercano en escenarios centrales o periféricos referidos a la escuela en las ciudades contemporáneas.

\section{Referencias Bibliográficas}

Alliaud, A. (2003). La biografía escolar en el desempeño profesional de los docentes noveles. (Tesis doctoral). Facultad de Filosofía y Letras, Universidad de Buenos Aires, Argentina. Recuperado de http://repositorio.filo.uba.ar/handle/filodigital/4100

Alliaud, A. y Antelo, E. (2011). Los gajes del oficio: enseñanza, pedagogía y formación. Buenos Aires: Aique.

André, C. (2009). Research practice and bibliographic information mapping supported by technological resources: impact on teacher education. (Tesis doctoral). Facultad de Educación, Universidad de São Paulo, São Paulo. doi:10.11606/T.48.2009.tde15122009-095048.

Recuperado de http://www.teses.usp.br/teses/disponiveis/48/48134/tde-15122009-095048/pt-br.php 
Cabeza, I. (2008). ¿Educación y territorio o territorialidad educativa? una alternativa para acercarnos a la enseñanza de la geografía y las ciencias sociales. II Encuentro de Ciencias Sociales y Humanas: "Narrativas, aprendizajes e identidades socioculturales". $1-5$.

Recuperado de http://1social.udistrital.edu.co:8080/documents/37512/40685/08.+Israel+Cabeza+\%26 + Daly+Acero.pdf?version $=1.0$

Córdoba, H. (2005). La escala local y la dimensión del lugar como alternativas para la enseñanza de la geografía escolar y la formación ciudadana. Revista Folios, 21, 83-89. Recuperado de https://www.redalyc.org/pdf/3459/345955978007.pdf

Mercado, R. y Moore, M. (1997). La geografía global como propuesta metodológica en la transformación curricular. VI Encuentro de Geógrafos de América Latina, Buenos Aires, Argentina. 1-8.

Moreno, N. (2011). Revisión de libros y novedades bibliográficas: Ciudades leídas, ciudades contadas. La ciudad como escenario didáctico para la enseñanza de la geografía. Revista Uni-pluri/versidad, 11(3). Recuperado de https://aprendeenlinea.udea.edu.co/revistas/index.php/unip/article/view/11843

Pastrán, R. (2007). Espacios fronterizos: una mirada desde la experiencia docente de pregrado. XI Encuentro de Geógrafos de América Latina Bogotá, Colombia.1-32.

Rodríguez, E., Moreno, N. y Cely, A. (2010). Geografía crítica y conocimiento social: Demandas de una geografía escolar renovada. En Moreno, N. y Hurtado, M. (Comps). Itinerarios geográficos en la escuela. Lectura desde la virtualidad (pp. 107-132). Recuperado de http://geopaideia.org/wp-content/uploads/2018/11/ITINERARIOSGEOGRAFICOS-EN-LA-ESCUELA-LECTURAS-DESDE-LA-VIRTUALIDAD.pdf

Rodríguez, E., Pérez, A. y Rodríguez, L. (2003). Algunas habilidades y procesos de pensamiento para comprender la dinámica espacial. Revista Conjeturas, 7, 70-86.

Rodríguez, E. y Von Prahl, A. (2008). Bogotá con ojos de taxista. En Rodríguez, C. y Moreno, N. (Comps). Cotidianidad y enseñanza geográfica (pp. 27-56). Bogotá: Editorial CÓDICE Ltda. 
Rodríguez, L., Arévalo, K., Barreto, J., Caselles, D., Chicangana, A., Delgadillo, A., Fajardo, A., Gómez, D., Hernández, J., Martínez, V., Mosquera, K., Sánchez, M., Trujillo, J. (2019). Matrices de resumen y contenido de mapeamiento informacional. Documento inédito. Bogotá: Universidad Distrital Francisco José de Caldas.

Santiago, J. (2013). La educación geográfica y el cambio pedagógico de su trabajo escolar cotidiano. Entorno Geográfico, 9, 8-27. Recuperado de http://revistas.univalle.edu.co/index.php/entornogeografico/article/view/3631

Soler, D., Rodríguez, L., Álvarez, D., Perico, S., Sánchez, C., Vásquez, W., Zambrano, B. (2018). Matrices de resumen y contenido de mapeamiento informacional. Documento inédito. Bogotá: Universidad Externado de Colombia y Universidad Distrital Francisco José de Caldas.

Schön, D. (1992). La formación de profesionales reflexivos. Hacia un nuevo diseño de la enseñanza y el aprendizaje en las profesiones. Barcelona: Paidós/MEC. 